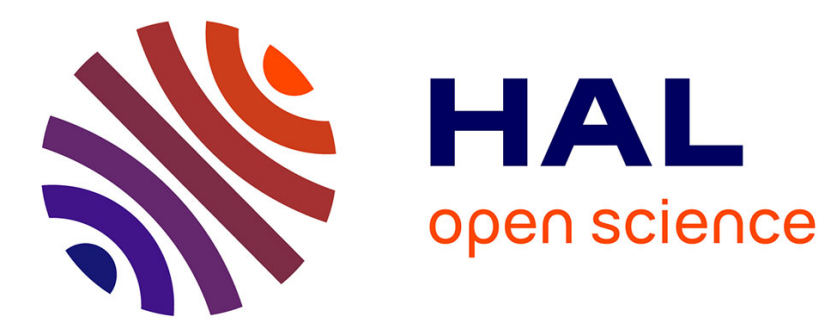

\title{
The danger of iteration methods
}

\author{
J. Villain, B. Séméria
}

\section{To cite this version:}

J. Villain, B. Séméria. The danger of iteration methods. Journal de Physique Lettres, 1983, 44 (22), pp.889-895. 10.1051/jphyslet:019830044022088900 . jpa-00232279

\section{HAL Id: jpa-00232279 https://hal.science/jpa-00232279}

Submitted on 1 Jan 1983

HAL is a multi-disciplinary open access archive for the deposit and dissemination of scientific research documents, whether they are published or not. The documents may come from teaching and research institutions in France or abroad, or from public or private research centers.
L'archive ouverte pluridisciplinaire HAL, est destinée au dépôt et à la diffusion de documents scientifiques de niveau recherche, publiés ou non, émanant des établissements d'enseignement et de recherche français ou étrangers, des laboratoires publics ou privés. 


\title{
LE JOURNAL DE PHYSIQUE-LETTRES
}

J. Physique - LETTRES 44 (1983) L-889 - L-895

15 NOVEMBRE 1983, PAGE L-889

Classification

Physics Abstracts

$03.20-64.00-61.70$

\section{The danger of iteration methods}

\author{
J. Villain and B. Séméria \\ Centre d'Etudes Nucléaires, Département de Recherche Fondamentale, \\ Laboratoire de Diffraction Neutronique, 85X, 38041 Grenoble Cedex, France
}

(Reçu le 5 septembre 1983, accepté le 5 octobre 1983)

\begin{abstract}
Résumé. - Si un hamiltonien $\mathscr{H}$ dépend de variables $\varphi_{i}$, les valeurs des variables qui le minimisent vérifient les équations $\partial \mathcal{H e} / \partial \varphi_{i}=0$. Si on résoud ce système d'équations par itération, il n'y a aucune raison pour que la solution obtenue soit celle qui minimise $\mathcal{H}$. Dans le cas d'un système harmonique dans un potentiel périodique par rapport aux $\varphi_{i}$, les fluctuations ont été calculées par Efetov et Larkin par la méthode d'itération. Le résultat est faux dans le cas d'un désordre fort. Même pour un désordre faible, il est faux pour un système unidimensionnel et pour un système fini de 2 particules. Nous suggérons que les résultats obtenus par itération sont faux dans tous les cas, et que, entre 2 et 4 dimensions, les fonctions de corrélation de paire de spins décroissent comme une puissance de la distance, en accord avec le résultat obtenu pour un autre modèle par Aharony et Pytte.

Abstract. - When a Hamiltonian $\mathcal{H}^{-}$depends on variables $\varphi_{i}$, the values of these variables which minimize $\mathscr{H}$ satisfy the equations $\partial \mathcal{H} / \partial \varphi_{i}=0$. If this set of equations is solved by iteration, there is no guarantee that the solution is the one which minimizes $\mathcal{H}$. In the case of a harmonic system with a random potential periodic with respect to the $\varphi_{i}$ 's, the fluctuations have been calculated by Efetov and Larkin by means of the iteration method. The result is wrong in the case of a strong disorder. Even in the weak disorder case, it is wrong for a one-dimensional system and for a finite system of 2 particles. It is argued that the results obtained by iteration are always wrong, and that between 2 and 4 dimensions, spin-pair correlation functions decay like powers of the distance, as found by Aharony and Pytte for another model.
\end{abstract}

\section{The harmonic model in a random field.}

Several authors [1-4] have studied the following Hamiltonian which constitutes an approximate description of an incommensurate phase with random, quenched impurities $[5,6]$

$$
\mathcal{H}=\frac{1}{4} \sum_{i, j=1}^{N} g_{i j}\left(\varphi_{i}-\varphi_{j}\right)^{2}-A \sum_{i=1}^{N} \cos \left(\varphi_{i}-\alpha_{i}\right)
$$


where the $\varphi_{i}$ 's denote $N$ continuous, real, dynamical variables. The $\alpha_{i}$ 's are $N$ independent random variables with a flat probability

$$
P\left(\alpha_{i}\right)=1 / 2 \pi \quad\left(-\pi<\alpha_{i} \leqslant \pi\right), \quad P\left(\alpha_{i}\right)=0 \text { otherwise } .
$$

Finally the $g_{i j}$ 's are given coefficients. For the sake of simplicity, the temperature may be assumed to be zero. Let $\left\langle\varphi_{i}-\varphi_{j}\right\rangle$ be the value of $\left(\varphi_{i}-\varphi_{j}\right)$ when $\mathcal{H}$ is minimized with respect to the $\varphi_{i}$ 's for given values of the $\alpha_{i}$ 's. The problem considered in this note is the evaluation (for $N$ fixed) of the ensemble averages

$$
\begin{gathered}
\overline{\left\langle\varphi_{i}-\varphi_{j}\right\rangle^{2}}=\left(\prod_{i} \int_{-\pi}^{\pi} \mathrm{d} \alpha_{i}\right)\left\langle\varphi_{i}-\varphi_{j}\right\rangle^{2} \\
\overline{\left\langle\cos \left(\varphi_{i}-\varphi_{j}\right)\right\rangle}=\left(\prod_{i} \int_{-\pi}^{\pi} \mathrm{d} \alpha_{i}\right)\left\langle\cos \left(\varphi_{i}-\varphi_{j}\right)\right\rangle .
\end{gathered}
$$

Minimization of (1) with respect to the $\varphi_{i}$ 's yields

$$
\sum_{j} g_{i j}\left(\varphi_{i}-\varphi_{j}\right)=A \sin \left(\alpha_{i}-\varphi_{i}\right)
$$

Hence

$$
\overline{\left\langle\varphi_{i}-\varphi_{j}\right\rangle^{2}}=A^{2} \sum_{l m}\left(G_{i l}-G_{j l}\right)\left(G_{i m}-G_{j m}\right) \overline{\left\langle\sin \left(\alpha_{l}-\varphi_{l}\right)\right\rangle\left\langle\sin \left(\alpha_{m}-\varphi_{m}\right)\right\rangle}
$$

where $G_{i j}$ is defined by

$$
\sum_{l}\left(\varepsilon \delta_{i l}+\delta_{i l} \sum_{m} g_{i m}-g_{i l}\right) G_{l j}=\delta_{i j} .
$$

The limit $\varepsilon \rightarrow 0$ is to be taken. Strictly speaking, equation $5 a$ has no solution for $\varepsilon=0$. The presence of $\varepsilon$ results from the addition of a term $\varepsilon \sum_{i} \varphi_{i}^{2} / 2$ to (1).

If $i$ denotes the sites of a periodic lattice and if the matrix $g_{i j}$ has the translation invariance of this lattice, the solution of $(5 a)$ is

$$
G_{l j}=\frac{1}{N} \sum_{q}[\mathcal{G}(0)-\mathcal{G}(q)+\varepsilon]^{-1} \exp \left(i q \cdot \mathbf{R}_{l j}\right)
$$

where $q$ denotes the points of the Brillouin zone and

$$
\mathcal{G}(q)=\sum_{j} g_{i j} \exp \left(i \mathbf{q} \cdot \mathbf{R}_{i j}\right)
$$

where $\mathbf{R}_{i j}$ is the vector joining sites $i$ and $j$.

As remarked by Efetov and Larkin [5], solutions of equations similar to (3) may be obtained by iteration. At order zero, one has

$$
\overline{\left\langle\sin \left(\varphi_{l}-\alpha_{l}\right)\right\rangle\left\langle\sin \left(\varphi_{m}-\alpha_{m}\right)\right\rangle} \stackrel{?}{=} \overline{\sin \alpha_{l} \sin \alpha_{m}}=\frac{1}{2} \delta_{l m} .
$$

The question mark recalls that the validity of this approximation is precisely the question to be discussed in this note. Insertion of $(6 a)$ into (4) yields

$$
\overline{\left\langle\varphi_{i}-\varphi_{j}\right\rangle^{2}} \stackrel{?}{=} \frac{1}{2} A^{2} \sum_{l}\left(G_{i l}-G_{j l}\right)^{2}
$$


Replacing $(2 b)$ by its first order cumulant expansion and using $(6 b)$, one obtains for any number $y$ the following equality

$$
\overline{\cos \left(y\left\langle\varphi_{i}-\varphi_{j}\right\rangle\right)} \stackrel{?}{=} \exp \left[-\frac{1}{4} A^{2} y^{2} \sum_{l}\left(G_{i l}-G_{j l}\right)^{2}\right]
$$

\section{The iteration solution.}

The iteration solution of an equation very similar to (3) has been studied by Efetov and Larkin [5], and it turns out [1] that their results are also valid for model (1). The essential result is that, if the iteration solution is accepted, relations 6 are exact.

Relation $6 c$ may be derived by the same method as relation 21 of reference 5 . The only differences are that i) reference 5 is restricted to dimension $D=3$. ii) a factor $y$ should be introduced for each external line when reading the diagrams. iii) each vertex with $p$ ingoing or outgoing lines contributes a factor

$$
A \frac{\mathrm{d}^{p}}{\mathrm{~d} \alpha_{j}^{p}} \sin \alpha_{j}
$$

As shown by Efetov and Larkin for $y=1$, diagrams arising from the left hand side of $(6 c)$ cancel, except those which have no external line, which yield the right hand side. This result holds for any value of $y$, because diagrams with a given number of external lines cancel separately. The same reason provides the justification of $(6 b)$, because the corresponding diagrams are just those which appear in $(6 c)$ with 2 external lines. Moreover, the cancellation noticed by Efetov and Larkin occurs before summation over vertices. This remark provides the proof of $(6 a)$, because the corresponding diagrams are those of $(6 b)$, with external lines reaching welldefined vertices $l, m$. The summation is over the other vertices only.

\section{Does iteration lead to the good solution?}

Equation 3 may have several solutions. Iteration leads to one of these solutions. The following example shows that it is not always the good solution, i.e. the one which minimizes (1). Let the system be crystalline and let the sample be parallelepipedic, with size $\mathbf{L}_{1} \times \mathbf{L}_{2} \times \cdots \times \mathbf{L}_{D}$. Let $\alpha_{j}$ have the form

$$
\alpha_{j}=\mathbf{q} \cdot \mathbf{R}_{j}
$$

where $q$ is such that $\cos \alpha_{j}$ and $\sin \alpha_{j}$ satisfy the usual periodic boundary conditions. The minimization of (1) has been solved in this case by Frank and Van der Merwe [9] in the continuum approximation. Neglecting boundary effects, the minimum energy $H$ has to satisfy the formula

$$
W / N g=(4 \kappa / k l) \int_{0}^{\pi} \mathrm{d} u \sqrt{1-k^{2} \sin ^{2} u}+\kappa^{2}\left(1-2 / k^{2}\right)-2 \pi q a / l+\frac{1}{2} q^{2} a^{2}
$$

where

$$
\kappa=\sqrt{A / g},
$$

$a$ is the interatomic distance, $k$ and $l$ are two parameters related by the formula

$$
k \int_{0}^{\pi} \mathrm{d} u / \sqrt{1-k^{2} \sin ^{2} u}=\kappa l
$$

and defined by the condition that the energy $(8 a)$ should be minimum.

The iteration solution considered by Efetov and Larkin corresponds to a vanishing average 
value of $\nabla \varphi$, i.e. $l=2 \pi / q a$. Indeed, it is seen from $(5 b)$ that $G_{i j}=G\left(\mathbf{R}_{i j}\right)$ satisfies periodic boundary conditions, $G\left(\mathbf{R}_{i j}\right)=G\left(\mathbf{R}_{i j}+\mathbf{L}_{\gamma}\right), \gamma=1,2, \ldots, D$. Since $\alpha_{i}$ and $\cos \alpha_{j}$ also satisfy periodic boundary conditions, these are also satisfied by the iteration solution, and this implies $l=2 \pi / q a$. This value does minimize ( $8 a$ ) for $A=0$. When $A$ is small, $\kappa$ and $k$ are small and expressions 8 may be expanded as power series of $k^{2}$. Elimination of $k$ and minimization with respect to $l$ for fixed $N$ yields

$$
l=(2 \pi / q a)\left[1+\pi\left(A / q^{2} a^{2} g\right)^{2}\right]+0\left(A^{3}\right) .
$$

Thus, the iteration solution (which corresponds to the first term alone) is not the good one for $A \neq 0$. For sufficiently large values of $\left(A / g q^{2} a^{2}\right)$, the good solution corresponds to $l=\infty-$ the commensurate solution of the Frank-van der Merwe problem [9].

Since the existing derivations of (6) are based on the iteration solution of (3), there is no reliable proof of (6).

\section{Relations 6 are wrong for strong disorder.}

However, one may argue that cases where the iteration method fails to give the correct solution of (3) may be exceptional, so that relations 6 can still be valid. In the present section, it will be shown that it is not the case, at least if $A$ is large.

For a large system, the average value

$$
\frac{1}{N} \sum_{l} \overline{\left\langle\sin \left(\varphi_{l}-\alpha_{l}\right)\right\rangle^{2}}
$$

is, with a probability about 1 , approximately equal to the ensemble average with an accuracy of order $1 / \sqrt{N}$. According to $(6 a)$, this ensemble average is $1 / 2$

$$
(1 / N) \sum_{l}\left\langle\sin \left(\varphi_{l}-\alpha_{l}\right)\right\rangle^{2} \stackrel{?}{=} 1 / 2
$$

This relation implies that the average value

$$
\frac{1}{N} \sum_{l}\left\langle\cos \left(\varphi_{l}-\alpha_{l}\right)\right\rangle
$$

cannot be very close to 1 . More precisely, the following inequality is not too difficult to derive from (9)

$$
\frac{1}{N} \sum_{l}\left\langle\cos \left(\varphi_{l}-\alpha_{l}\right)\right\rangle \stackrel{?}{<} 0.95
$$

If one considers a state satisfying (9), its energy $W_{1}$ has, according to (1) and (10), a probability close to 1 to satisfy the relation

$$
W_{1}>-0.95 A N \text {. }
$$

On the other hand, the energy $W_{0}$ of the state $\varphi_{i}=\alpha_{i}$ satisfies the inequality

$$
W_{0}<-A N+2 g \pi^{2} N \text {. }
$$

$W_{0}$ is lower than $W_{1}$ if

$$
A>40 g \pi^{2} \text {. }
$$


Thus, if inequality 13 is true, the ground state of a very large system has a very low probability to satisfy (9). Relations 9 and $6 a$ are therefore wrong. Relations $6 b$ and $c$, which also rely on the iteration solution, are probably wrong too.

\section{Relations 6 are wrong for a linear chain.}

Relation $6 b$ will now be shown to be wrong in the case of a long linear chain with interactions $g_{j, j+1}=g$ between nearest neighbours. Indeed, $(6 b)$ is in contradiction with the exact inequality

$$
\left|\left\langle\varphi_{j+1}-\varphi_{j}\right\rangle\right| \leqslant \pi
$$

which holds at $T=0$. Indeed, if a state does not satisfy (14) for some value of $j$, it is not a ground state because the energy can be lowered by shifting all $\varphi_{i}^{\prime}$ 's by $2 \pi($ or $-2 \pi)$ for $i>j$. Relation 14 implies

$$
\overline{\left\langle\varphi_{j+r}-\varphi_{j}\right\rangle^{2}}<\pi^{2}|r| \text {. }
$$

On the other hand, the Green function $(5 b)$ is, for $\left|R_{l j}\right| \ll N$

$$
\begin{aligned}
G_{l j} & =(1 / 4 \pi g) \int_{-\pi}^{\pi} \mathrm{d} q\left(\cos q R_{l j}\right) /(1-\cos q+\varepsilon / 2 g) \\
& =(1 / 4 \pi) \sqrt{2 g \varepsilon} \exp \left(-\left|R_{l j}\right| \sqrt{\varepsilon / 2 g}\right) .
\end{aligned}
$$

Insertion into $(6 b)$ yields

$$
\left\langle\varphi_{i}-\varphi_{j}\right\rangle^{2} \stackrel{?}{=} \frac{1}{2}\left(A R_{i j} / 4 \pi g\right)^{2} \sum_{l} \exp \left(-\left|R_{l j}\right| \sqrt{2 \varepsilon / g}\right) .
$$

In the limit $\varepsilon=0$, this expression goes to infinity with $N$, in contrast with the exact relation 15 . Therefore, relation $6 b$ is wrong for a large, one-dimensional system, except if $A=0$.

\section{The case $N=2$.}

It is of interest to treat the simple case of a set of two particles. Hamiltonian (1) may be written as

$$
\mathscr{H}=\frac{g}{2}\left(\varphi_{1}-\varphi_{2}\right)^{2}-2 A \cos \frac{1}{2}\left(\varphi_{1}-\varphi_{2}-\alpha_{1}+\alpha_{2}\right) \cos \frac{1}{2}\left(\varphi_{1}+\varphi_{2}-\alpha_{1}-\alpha_{2}\right) .
$$

If this expression is maximum, the second cosine should be maximum or minimum (according to the sign of the other cosine) since the combination $\left(\varphi_{1}+\varphi_{2}\right)$ does not appear elsewhere. This implies

$$
\varphi_{1}+\varphi_{2}=\alpha_{1}+\alpha_{2}+2 n \pi
$$

where $n$ is an integer. Inserting (9) into (3), one obtains

where

$$
\sin \psi=-2(-1)^{n} \frac{g}{A}(\psi+\alpha / 2)
$$

and

$$
\psi=\varphi_{1}-\alpha_{1}-n \pi
$$

$$
\alpha=\alpha_{1}-\alpha_{2} .
$$


The energy $E$ corresponding to a solution of (10) is

$$
E=2 g(\psi+\alpha / 2)^{2}-2 A(-1)^{n} \cos \psi .
$$

Using, for instance, the graphical solution of (11), it may be shown that the ground state corresponds to even values of $n$ if $\cos \alpha / 2>0$, and to odd values of $n$ if $\cos \alpha / 2<0$. As a matter of fact, it is always possible to redefine $\alpha_{1}$ or $\alpha_{2}$ in such a way that

$$
-\pi<\alpha<\pi \text {. }
$$

Indeed, if this condition is not satisfied, it can be fulfilled after addition of an appropriate term $\pm 2 \pi$ to $\alpha_{1}$ or $\alpha_{2}$. With this convention, the probability $P(\alpha) \mathrm{d} \alpha$ that $\alpha$ lies in an interval $\mathrm{d} \alpha$ is given by

$$
\begin{aligned}
& P(\alpha)=1 / 2 \text { if }-\pi<\alpha<\pi \\
& P(\alpha)=0 \text { otherwise. }
\end{aligned}
$$

The expansion of (4) to fourth order in $A$ can easily be obtained from the iteration of (10). One finds

$$
\begin{aligned}
\overline{\left\langle\varphi_{1}-\varphi_{2}\right\rangle^{2}}=\left(A^{2} / g^{2}\right) \overline{\sin ^{2} \alpha / 2}-\left|A^{3} / g^{3}\right| \overline{\sin ^{2}(\alpha / 2) \cos (\alpha / 2)}- & \\
& -\frac{1}{4}(A / g)^{4} \overline{\left(\sin ^{4} \alpha / 2\right.}-3 \overline{\left.\sin ^{2} \alpha / 2 \cos ^{2} \alpha / 2\right)}+\cdots
\end{aligned}
$$

The average values are easily calculated from (23) and the result is

$$
\left\langle\varphi_{1}-\varphi_{2}\right\rangle^{2}=\frac{1}{2}(A / g)^{2}-(2 / 3 \pi)|A / g|^{3}+0\left(A^{5} / g^{5}\right) .
$$

Even terms vanish as predicted by Efetov and Larkin [5]. But odd terms do not vanish. Note that the first term of (24) is twice as large as in $(6 b)$. This discrepancy is a finite size effect which should become smaller and smaller when $N$ increases. However, the non-analytic character of (24) is striking, when compared with the simple analytic result $(6 b)$.

\section{Conclusion.}

For a finite system with $N=2$, the average value $\overline{\left\langle\varphi_{i}-\varphi_{j}\right\rangle^{2}}$ is non-analytic in $A$ at $A=0$. If the analytic formula $6 b$ is valid for an infinite system, there should be a phase transition, and therefore a non-analyticity for some value $A_{\mathrm{c}}$. The simplest possibility is that the non-analyticity always takes place at $A=0$. This is probably the case for $D=1$, since formula $6 b$ is certainly wrong in this case as seen in section 5 .

This possibility is also suggested by a renormalization group calculation [6] which predicts, for $2<D<4$,

$$
\overline{\left\langle\cos \left(\varphi_{i}-\varphi_{j}\right)\right\rangle} \sim\left|R_{i j}\right|^{-\tau}
$$

while relation $6 b$ yields an exponential decay. The result $(25)$ has been previously obtained by Aharony and Pytte [7, 8] for a model in which, in contrast with model (1), vortices are not excluded. According to reference 1, vortices are irrelevant. The exponent $\tau$ obtained in reference 6 vanishes for $D=4$, in contrast with references 7 and 8 .

For $D>4$, the iteration method and the renormalization group approach agree to predict that long range order is not destroyed by the random potential. Nevertheless, relation $6 a$ is 
wrong for large $A$, and there is no reason to believe it when $A$ is small. This fact has no spectacular qualitative consequences, in contrast with what happens below 4 dimensions.

\section{Acknowledgments.}

It is a pleasure to thank Philippe Nozières for critical reading of a part of the manuscript, and Mirta Gordon, for useful discussions.

\section{References}

[1] Dotsenko, V. S., Feigelman, M. V., J. Phys. C 16 (1983) L-803.

[2] Chudnovsky, E. M., Serota, R. A., Phys. Rev. B 26 (1982) 2697.

[3] Efetov, K. B., Sov. Phys. JETP 48 (1978) 949; Zh. Eksp. Teor. Fiz. 75 (1978) 1885.

[4] Cardy, J. L., Ostlund, S., Phys. Rev. B 25 (1982) 6899.

[5] Efetov, K. B., LaRKIN, A. I., Zh. Eksp. Teor. Fiz. 72 (1977) 2350 ; Sov. Phys. JETP 45 (1977) 1236.

[6] Villain, J., Fernandez, J., to be published in Z. Phys.

[7] Aharony, A., Pytte, E., Phys. Rev. Lett. 45 (1980) 1583.

[8] Aharony, A., J. Phys. C 14 (1981) L-841.

[9] Frank, F. C., van Der Merwe, J. H., Proc, R. Soc. 198 (1949) 205. Formulae given here correspond to a problem where $N$ has a fixed value, as is the case for an $X Y$ magnet. The situation is different in the case of adsorption at fixed pressure, but the extension to this case is straightforward and the qualitative conclusions are the same. 